Bull. Mater. Sci., Vol. 6, No. 2, May 1984, pp. 243-258. (C) Printed in India.

\title{
Electrical properties of polycrystalline silicon and zinc oxide semiconductors
}

\author{
S N SINGH, S KUMARI and B K DAS \\ National Physical Laboratory, New Delhi 110 012, India. \\ Abstract. Polycrystalline silicon and zinc oxide ceramic are important electronic materials. \\ The electrical properties which determine the applications of polycrystalline silicon in \\ integrated circuits and solar cells and that of $\mathrm{ZnO}$ ceramic in varistors are due primarily to \\ grain boundary effects in them. A large amount of information in this area has already been \\ gathered in literature but the quantitative understanding of grain boundary effects in these \\ materials is not yet complete. In this review the important aspects of grain boundaries and their \\ effects on transport and photoelectric properties of polycrystalline silicon and on the I-V \\ characteristic of $\mathrm{ZnO}$ varistors are discussed.
}

Keywords. Polycrystalline semiconductors; electrical properties; grain boundary effects; polysilion; $\mathrm{ZnO}$ varistors.

\section{Introduction}

The intergranular regions of polycrystalline semiconductors often affect the electrical properties of the solids in such a way that they determine the applications of these solids. There are several ways this can arise. Grain boundaries (GBS) are twodimensional imperfections containing highly complex structural defects and in most cases are electrically active. Impurity atoms also segregate at the grain boundaries. Any of these conditions can lead to the formation of a space charge layer near the grain boundaries. In case of a metal, this is of such narrow spatial extent that it is best treated in terms of a scattering formalism. In an insulator, the extent of these space charge regions is large and the influence of GB $S$ are better viewed as large perturbations on the bulk Fermi levels. The intermediate case of polycrystalline semiconductors is of considerable technological importance. This is an attempt to review the important properties of grain boundaries and the grain boundary and doping effects on the transport and photovoltaic properties of some semiconductors like silicon and zinc oxide and the methods employed to determine these effects.

\section{Polycrystalline silicon}

The many emerging applications of polycrystalline silicon (polysilicon) including discrete devices and integrated circuits have prompted several recent studies of carrier transport in polysilicon. The role of these GBS on the photovoltaic properties of inexpensive polycrystalline solar cells has also received considerable attention by various research groups. 


\subsection{Grain boundary effects in polycrystalline silicon}

2.1a Impurity segregation: The first direct physical evidence of segregation of impurities at GBS in cast and directionally solidified polysilicon was gathered by Kazmerski et al (1980). Using scanning Auger microscopy (SAM) and secondary ion mass spectroscopy (sIMs) techniques, they showed that much larger concentrations of $\mathrm{Fe}, \mathrm{Ti}, \mathrm{Co}, \mathrm{Ni}, \mathrm{Cn}, \mathrm{Zn}, \mathrm{Sb}, \mathrm{C}, \mathrm{Al}$ and $\mathrm{Mg}$ are present at the grain boundaries as compared to the grain interior. Similar experiments (Kumari et al 1983) on grain boundaries of polysilicon of solar grade purity also confirmed the grain boundary enrichment as shown in figure 1. In a different experiment, Pollock et al (1982) carried out $\mathrm{Cu}$ and $\mathrm{P}$ diffusions into cast polysilicon and studied $\mathrm{GB}$ enrichment with $\mathrm{Cu}, \mathrm{H}, \mathrm{C}$ and $\mathrm{O}$ by the sIm technique. They showed that $\mathrm{H}, \mathrm{C}$ and $\mathrm{O}$ accumulate at GBs (figure $2 \mathrm{a}$ ). A secondary ion image of $\mathrm{Cu}$ obtained using oxygen ion bombardment shown in figure $2 \mathrm{~b}$ indicates that large clusters of $\mathrm{Cu}$ are present along $\mathrm{GB}$ s. Segregation of impurities at GB S particularly those giving deep levels can significantly modify the electrical activity of the GBS. This complicates the understanding of the role of GB $\mathrm{S}$ in influencing the majority and minority carrier phenomena in polysilicon.

2.1b Enhanced impurity diffusion: The diffusion coefficient of impurity is higher in the GBS than in the grains (Matre 1971) and consequently the diffusion of the impurities along the GBS is deeper. This in conjunction with the preferential segregation of impurities at GBS can considerably change the picture of junction formation in polysilicon devices than in single crystalline devices. For example, in polysilicon solar cells, wilile the enhanced diffusion of dopants along GBS makes the $p-n$ junction interface irregular, the enhanced diffusion coupled with the tendency of the irnpurities to segregate preferentially at $\mathrm{GBS}$ reduces the leakage resistance of the junction considerably leading to degradation of the I-V characteristics of polysilicon solar cells (Lindmayer 1978). Figure 3 shows a schematic diagram of an irregular $p$ - $n$ junction formed due to the GB S as mentioned above and figure 4 depicts a SEM picture as a direct evidence of deeper diffusion of dopant impurity at GBS (Jain et al 1980a). Figure 5 shows how this results in a greater effective junction depth in a small grain polysilicon (Jain et al 1981).

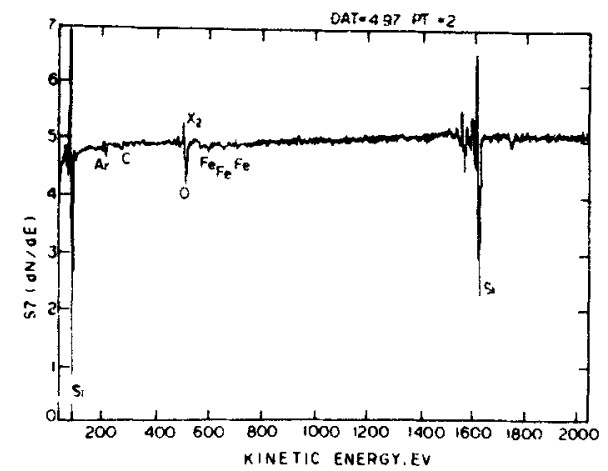

(a)

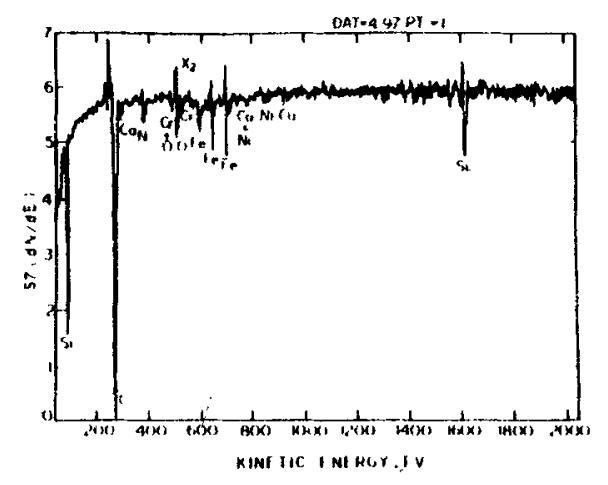

(b)

Figure 1. Auger spectrum taken in a. grain region and b. GB on a fractured surface of solar grade polysilicon (after Kumari et al 1982). 

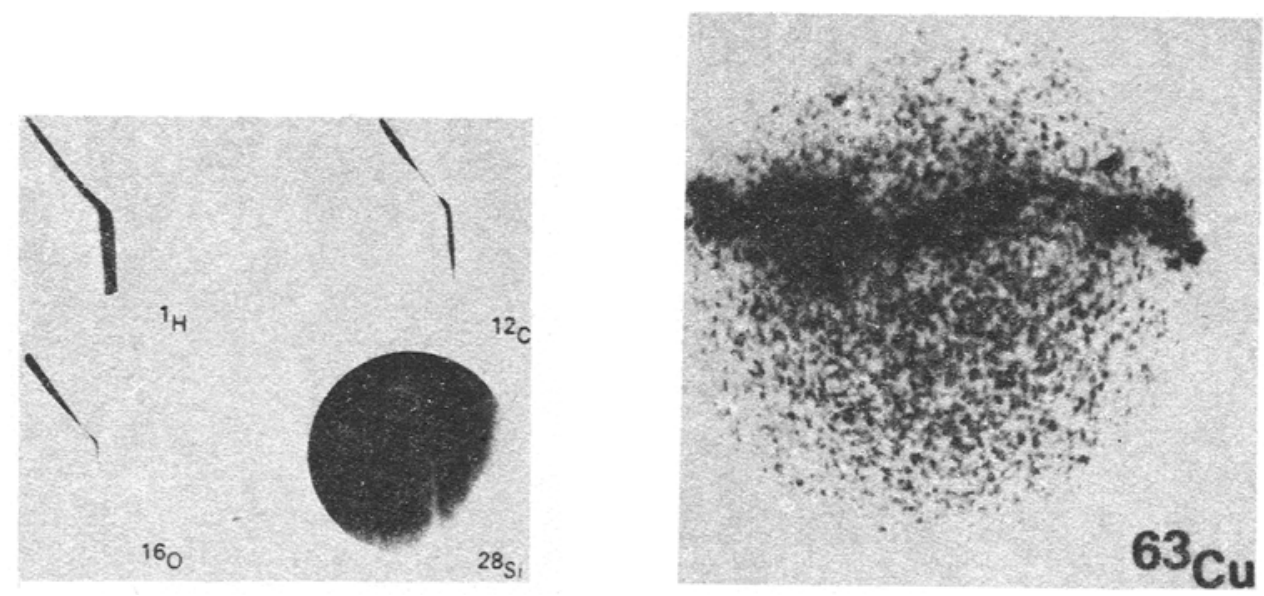

Figure 2. a. Secondary ion images of $\mathrm{H}, \mathrm{C}, \mathrm{O}$ and $\mathrm{Si}$ obtained using cesium ion bombardment of $\mathrm{Cu}$ diffused polycrystalline silicon wafer. b. An image of $\mathrm{Cu}$ distribution in another area of the above sample (after Pollock et al 1982).

2.1c Majority carrier trapping: The presence of the defects at grain boundary gives rise to energy states in the forbidden gap (Card and Yang 1977). These states commonly referred to as GB states or the interface states trap majority carriers from the surrounding grains. This decreases the free carrier density in the bulk polysilicon and creates a space charge (or depletion) layer on both sides of a grain boundary (in onedimensional model). The space charge layer in turn results in the creation of potential barrier at the GB. The nature of the potential barrier is such that it opposes the flow of majority carriers from one grain to the other and favours the flow of minority carriers towards the grain boundary. The band bending at a GB for $n$-type semiconductor under thermal equilibrium is shown in figure 6. Here the grains have been assumed to be identical. In the figure $d_{L}=d_{R}$, at equilibrium, if the grains are identical both structurally and electrically. The difference in the doping level of the grains can result in the asymmetrical band bending. The decrease in the free carrier density in the grains due to their trapping at GB $S$ and the existence of the grain boundary potential barriers, as will be discussed a little later, can have profound effect on the electrical behaviour of poly-silicon.

Equating the depletion layer charge $Q_{d}$ to the trapped charge $Q_{T}$ in the GB s, the height of the potential barrier can be easily calculated. For the depletion layer approximation, the solution of Poisson's equation gives (Seager and Castner 1978)

$$
Q_{d}=\left(8 \varepsilon N_{D} q \phi_{B}\right)^{1 / 2},
$$

Here, $\varepsilon$ is the dielectric constant of silicon, $q$ is the electronic charge and $N_{D}$ is the doping concentration in the grain (in the present case as $n$-type). For determination of $Q_{T}$, however, the knowledge of the nature of the energy level of the interface states $(\mathrm{eg}$. whether the states are distributed uniformly throughout the forbidden gap or they have a delta function distribution or some other distribution) is essential. Unfortunately the picture of the energy levels of the GB states is not yet clear. Poon and Hwang (1982) have developed a phenomenological model of grain boundary trapping states in polysilicon 


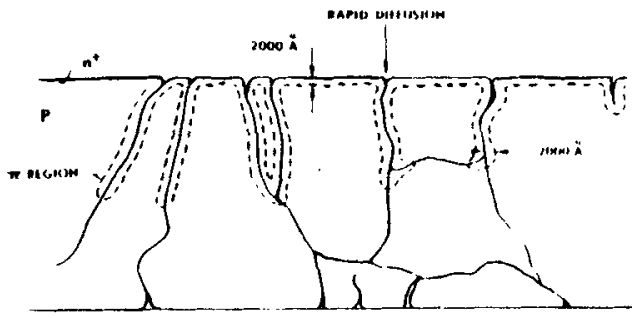

Figure 3. Schematic cross sectional view of diffused semicrystalline structure showing deeper diffusion along the GBs.

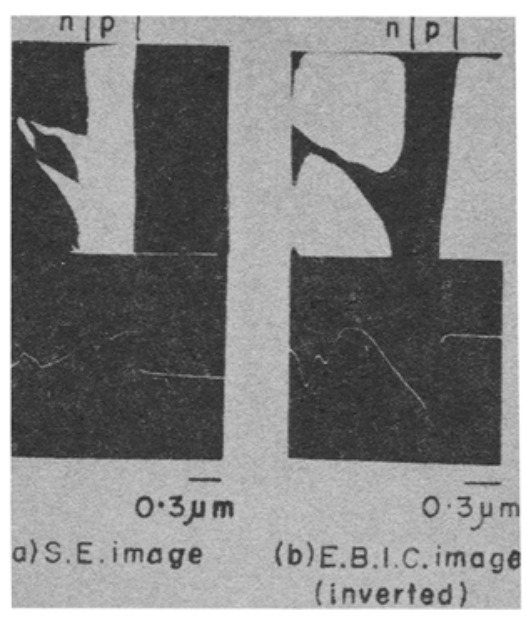

Figure 4. a. Secondary emission (SE) and b. Electron beam induced current (EBIC) images at the cleaved surface of a polycrystalline silicon solar cell (after Jain et al 1981).

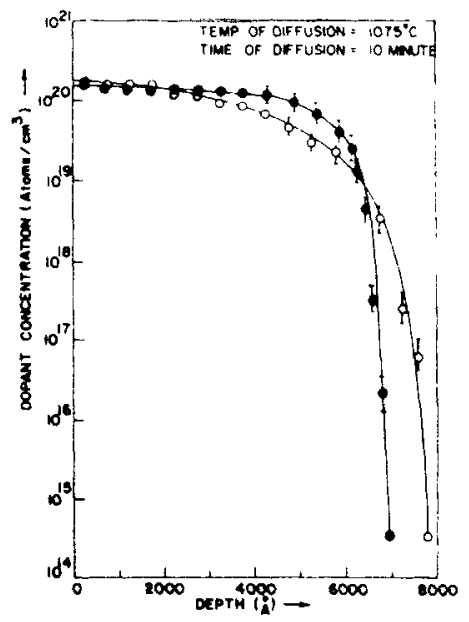

Figure 5. Dopant profile of boron in solar grade poly (o) and single (๑) crystalline silicon (after Jain et al 1981).

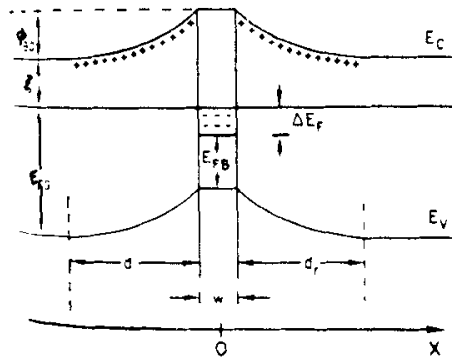

Figure 6. Energy band diagram for two grains and their grain boundary region in an $n$-type polysilicon. $\left(E_{F G}\right.$-Fermi energy level in the bulk of the grain with valence band edge as the reference; $E_{F D}$-the neutral $E_{v}$ Fermi level at the GB; $\phi_{B}$ height of the grain boundary potential barrier; $d_{L}$ and $d_{R}$ the space charge layer widths in the left and right grain respectively). 
under optical illumination. They find discrete ( $\delta$-function) distribution to be inappropriate. However, many experimental data on dark conductivity and Hall mobility agree with $\delta$-function distribution assumption excellently (Kishore et al 1983; Baccarani et al 1978). For uniform distribution of interface states in the band-gap $Q_{T}$ can be computed as

$$
Q_{T}=q N_{\text {is }}\left(E_{F G}-E_{F B}-q \phi_{B}\right) .
$$

For this case $\phi_{B}$ can be determined by equating (1) and (2)

where $\quad \delta=\frac{1}{q}\left(E_{F G}-E_{F B}\right)$,

$$
\phi_{B}=\delta\left[1+\frac{\alpha}{2 \delta}-\left\{\frac{\alpha}{\delta}+\left(\frac{\alpha}{2 \delta}\right)^{2}\right\}^{1 / 2}\right],
$$

and

$$
\alpha=\frac{8 \varepsilon N_{D}}{q^{3} N_{\text {is }}^{2}} .
$$

Discrete energy level, on the other hand, is obtained from the solution of equation (Martinez and Piqueras 1980).

$$
\begin{aligned}
\left(\frac{8 \varepsilon N_{D} \phi_{B}}{q^{2}}\right)^{1 / 2}= & q N_{\mathrm{is}}\left[\frac{1}{1+\exp \left[\left(E_{T}-E_{F G}-q \phi_{B}\right) / k T\right]}\right. \\
& \left.-\frac{1}{1+\exp \left[\left(E_{T}-E_{F B}\right) / k T\right]}\right]
\end{aligned}
$$

where $E_{T}$ is the energy of the trap level and $N_{\text {is }}$ is the density of interface states per unit area. In the further discussion (3) and (4) will be used to describe some majority carrier phenomena in polysilicon.

2.1d Enhanced minority carrier recombination: The GB states act not only as trap centres for majority carriers but also as efficient recombination centres for minority carriers. The minority carrier recombination at $\mathrm{GB}$ is larger if there exists potential barrier at the GB. Following Card and Yang (1977) the minority carrier lifetime in the space charge region of $n$-type polysilicon denoted as $\tau_{g b}$ is given by

$$
\tau_{g b}=\frac{2 \mathrm{~d} \exp \left(-q \phi_{B} / k T\right)}{3 \sigma v N_{\text {is }}\left(E_{f n}-E_{f p}\right)},
$$

where $\phi_{B}$ is the barrier height, $d$ is grain size, $\sigma$ is the capture cross-section, $v$ is the thermal velocity of carrier, $N_{\text {is }}$ is the interface state density and $E_{f_{n}}$ and $E_{f p}$ are the quasi Fermi levels for electrons and holes respectively. The equation is valid for quasi Fermi level for minority carriers remaining flat across the GB. The assumption usually holds for low level conditions unless the recombination velocity of minority carriers $(S)$ at the edge of the depletion layer is infinitely high. However, according to Fossum and Lindholm (1980) $\tau_{g b}$ is given by

$$
\tau_{g b}=2 D b_{0}^{2},
$$

where $D$ is the diffusion coefficient of the minority carriers in the grains and $b_{0}$ is related with $d, D$ and $S$ by the following equation

$$
b_{0} \tan \left(b_{0} d / 2\right)=S / D \text {. }
$$


If $S \rightarrow \infty, b_{0}=\pi / d$ and, then,

$$
\tau_{g b}=2 \pi^{2} D / d^{2} \text {. }
$$

For this case the effective lifetime $\tau_{\text {eff }}$ is given by

$$
\frac{1}{\tau_{\mathrm{eff}}}=\frac{1}{\tau_{n}}+\frac{2 \pi D^{2}}{d^{2}},
$$

where $\tau_{n}$ is the lifetime in the grains.

Assuming $D=25 \mathrm{~cm}^{2} / \mathrm{sec}$ the effective lifetime of electrons in $p$-type polysilicon was calculated as a function of $d$ and is depicted in figure 7. For smaller values of $d, \tau_{\text {eff }}<\tau_{n}$ but $\tau_{\text {eff }} \rightarrow \tau_{n}$ for $d$ greater than a certain value $d_{c}$, which is smaller for a smaller $\tau_{n}$. The performance of a solar cell made on polysilicon, in which the grain boundary activities is high $(S \rightarrow \infty)$ is highly limited by its grain size if $d<d_{c}$.

\subsection{Transport properties of polycrystalline silicon}

Current conduction process in polysilicon is significantly affected due to the presence of the grain boundary potential barriers. Thermionic emission (TE) theory has been applied by many workers [Seto 1975; Baccarani et al 1978; Martinez and Piqueras 1980; Kumar and Satyam 1981] to describe the current conduction in polysilicon. According to TE theory, the current density $J$ for an applied voltage $V$ across the barrier is given by

$$
J=q n_{g}\left(k T / 2 \pi m^{*}\right)^{1 / 2} \exp \left(-q \phi_{B} / k T\right)[\exp (q V / k T)-1],
$$

where $n_{g}$ is the free majority carrier density in the grain and $m^{*}$ is the effective mass of the majority carrier. Equation (10) shows that the $J-V$ characteristic of a polysilicon

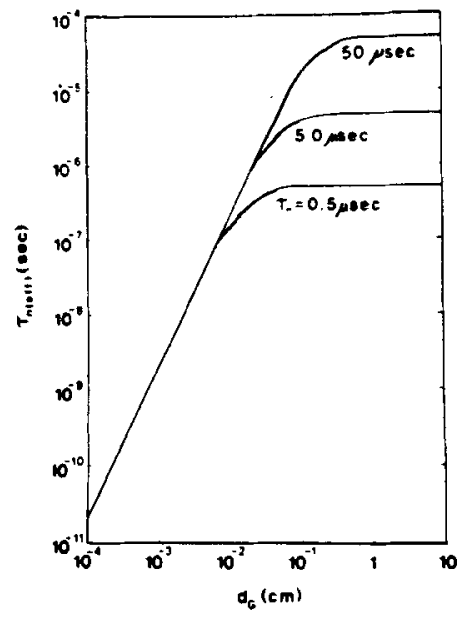

Figure 7. Effective minority carrier life time as a function of grain size for a p-type polysilicon (after Fossum and Lindholm 1980)

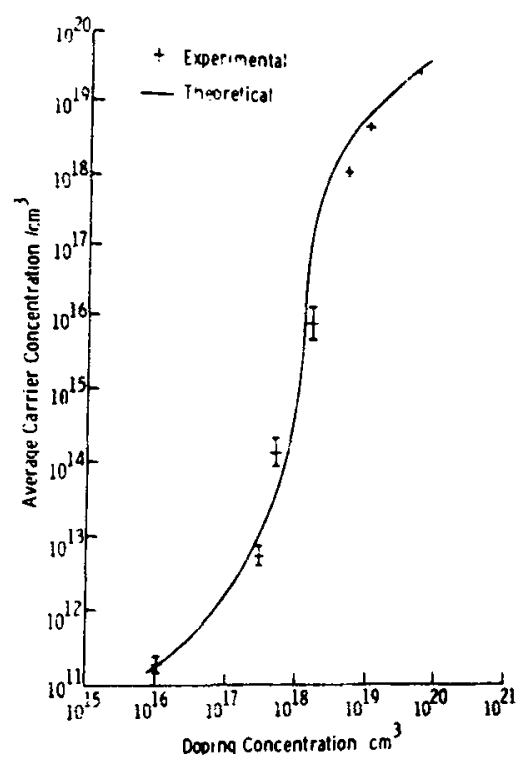

Figure 8. Average carrier concentration vs doping concentration for a polysilicon film at room temperature (grain size 200 A) (after Seto 1975). 
specimen is non linear unless the applied voltage drop per GB $V \ll k T / q$. In the ohmic regime $(V \ll k T / q)$, the $G B$ potential barrier can have a profound influence on the transport properties of polysilicon. In the following we will discuss GB effect on free carrier density, majority carrier mobility and conductivity of polysilicon.

2.2a Free carrier density: The GB states as discussed earlier trap majority carriers from the surrounding grains. The trapping decreases the density of free carriers. The decrease is more, if the interface state density is large, the doping in the grain is small and more significantly if the grain size is small. For very small grain sizes, almost the entire grain regions can be made devoid of the extrinsic carriers. Owing to this reason in small polysilicon, the free carrier density is far too low than the doping concentration $(N)$ unless $N$ becomes larger than a certain value $N_{c}$ which will be a function of $N_{\text {is }}$ and $d$. Figure 8 shows the average carrier density $v s$ doping concentration for a polysilicon film $(d \sim 200 \AA)$ at room temperature after Seto (1975). The theoretical curve was obtained on the basis of carrier trapping at GBS.

2.2b Majority carrier mobility: The GB potential barrier opposes the flow of majority carriers across it and thus effectively the majority carrier mobility is reduced. Many workers have applied TE theory in conjunction with carrier trapping at GB to derive an expression for the effective majority carrier mobility $\mu_{\mathrm{eff}}$. For the case of partially depleted grain Seto (1975) obtained

$$
\mu_{\mathrm{eir}}=\frac{q d}{\left(2 \pi m^{*} k T\right)^{1 / 2}} \exp \left(-q \phi_{B} / k T\right)
$$

This expression ignores the contribution of grain to $\mu_{\mathrm{eff}}$. Kumar and Satyam's (1981) expression for $\mu_{\mathrm{eff}}$ is

$$
\mu_{\mathrm{eff}}=\frac{\mu_{g}}{\left(1-n_{t} t / N_{D} d\right)+\frac{\mu_{g}}{s q d}\left(2 \pi m^{*} k T\right)^{1 / 2} \exp \left(q \phi_{B} / k T\right)} .
$$

Here $n_{t}$ is the density of filled states per unit volume, $t$ is the physical thickness of GB, $\mu_{g}$ is the mobility in the grain and $s$ is a fitting constant. There have been a few attempts to explain the mobility (Colinge et al 1981; Kim et al 1982) data using diffusion theory of current transport also. Recently Kishore et al (1983) have derived a rather general expression for $\mu_{\text {eff }}$, which is based on the thermionic-emission-diffusion theory of Crowell and Sze (1966). The expression is

$$
\mu_{\mathrm{eff}}=\frac{\mu_{g}}{\left(1-\frac{n_{t} t}{N_{D} d}\right)+\frac{k T}{q} \frac{\mu_{g}}{d}\left(\frac{1}{v_{D}}+\frac{1}{v_{R}}\right) \exp \left(\frac{q \phi_{B}}{k T}\right)},
$$

where $\quad v_{D}=\mu_{g} E_{B}$.

and $\quad v_{R}=\left(k T / 2 \pi m^{*}\right)^{1 / 2}$.

Here $E_{B}$ is the field near the potential maximum. For $v_{D} \gg v_{R}$ (13) reduces to (12), neglecting the presence of $s$, and it will reduce to (11) if $v_{D} \gg \mathrm{v}_{R}$ and the grain size is sufficiently small. All equations (11)-(13) show that $\mu_{\text {eff }}$ is small if $d$ is small and it increases with $d$. Equations (12)-(13) show that for very large value of $d, \mu_{\mathrm{eff}} \rightarrow \mu_{g}$. It is also noted that $\mu_{\mathrm{eff}}$ is less for a higher $\phi_{B}$. Decrease in $\phi_{B}$ increases $\mu_{\mathrm{eff}}$, which tries to 
approach $\mu_{g}$. Under identical conditions, the adverse effect of $\phi_{B}$ on $\mu_{\text {eff }}$ will be more pronounced, if $d$ is small.

2.2c Conductivity: The effect of $\mathrm{GB}$ on the free carrier density and carrier mobility results in a decrease in the conductivity $(\sigma)$ of polysilicon. Using (13), $\sigma$ can be expressed as

$$
\sigma=\frac{n_{g} q \mu_{g}}{\left(1-\frac{n_{t} t}{N_{D} d}\right)+\frac{k T}{2} \frac{\mu g}{d}\left(\frac{1}{v_{D}}+\frac{1}{v_{R}}\right) \exp \left(\frac{q \phi_{B}}{k T}\right)}
$$

where $n_{g}$ is the free carrier density in the grain. Equation (14) represents a general case. However, in many cases, in practice, $\phi_{B}$ is large and $d$ is small such that the carrier mobility is given by (11). In such a case the conductivity will be given by

$$
\sigma=\frac{q^{2} n_{g} d}{\left(2 \pi m^{*} k T\right)^{1 / 2}} \exp \left(-\frac{q \phi_{B}}{k T}\right) .
$$

Substituting the value of $n_{g}$ from equation

$$
n_{g}=2\left(\frac{2 \pi m^{*} k T}{h^{2}}\right)^{3 / 2} \exp (-\xi / k T),
$$

into (15) we obtain,

$$
\sigma=\frac{4 \pi m^{*} k T q^{2} d}{h^{3}} \exp \left(-E_{o} / k T\right),
$$

where $E_{\sigma}=q \phi_{B}+\xi ; \xi$ being the difference in the energies of the conduction band edge and the Fermi level in the grain.

A comparison of (9) and (15) shows that the activation energy of $\mu_{\mathrm{eff}}$ and $\sigma$ are not identical. Gaef et al (1979) have shown that $E_{\sigma}$ is practically independent of $T$. This is in contrast with $\phi_{B}$ which, as seen from (3) or (4), depends on $E_{F G}$ and therefore decreases with $T$ owing to the shifting of $E_{F G}$ towards the intrinsic Fermi level at a higher $T$.

\subsection{Photoelectric properties of polysilicon}

2.3a. Photovoltaic properties: Large grain $(d \sim 1 \mathrm{~mm}$ or larger) polysilicon has been finding considerable attention due to its potential as low cost material for terrestrial solar cells. The grain boundaries have deleterious effect on the performance of polysilicon solar cells. GBS decrease the short circuit current density $\left(J_{\mathrm{sc}}\right)$, open circuit voltage $\left(V_{\text {oc }}\right)$ shunt resistance $\left(K_{\mathrm{sh}}\right)$ and increase the ideality factor $(n)$ and reverse saturation current density $\left(J_{0}\right)$ of the solar cell. The effect of $J_{\mathrm{sc}}$ is directly related to the enhanced minority carrier recombination at GBS. In the conventional solar cell design, the $p-n$ junction is a plane perpendicular to the cell thickness and is situated at $\leqslant 0.5 \mu \mathrm{m}$ below the front surface. In this design the carriers to be collected by the $p-n$ junction travel along the cell thickness. The effect of GBS on $J_{s c}$ is more severe, if the carriers have to cross GBs before they could be collected. The effect of GBS is minimised if the grains are columnar along the cell thickness and therefore in practice polysilicon cells are generally made on columnar grain polysilicon material. The deeper diffusion of the dopant impurity at GB effectively increases the area of the space charge at the $p-n$ junction. This enhances the space charge recombination, which in turn increases $n$ and 
$J_{0}$. As a result $n$ and $J_{0}$ increase with the decrease in grain size. A combination of effects of $\mathrm{GB}$ on $J_{\mathrm{sc}}, n$ and $J_{0}$ leads to decrease in $V_{\mathrm{oc}}$. Many workers (Fischer and Pschunder 1977; Jain et al 1980b) have studied the effect of GBS on the performance of columnar grain silicon solar cells. Representing the $J-V$, characteristic by equation.

$$
J=J_{\mathrm{sc}}-J_{01}\left(\exp \frac{q V_{J}}{n_{1} k T}-1\right)-J_{02}\left(\exp \frac{q V_{J}}{n_{2} k T}-1\right)-\frac{V_{J}}{R_{\mathrm{sh}}}
$$

where the second and third terms on the right side represent respectively the contributions of bulk recombination and space charge recombination. Kumari et al (1981) have studied the effect of grain size on the photovoltaic properties of $P^{+} N$ polysilicon solar cells. Effect of grain size on $V_{\mathrm{oc}}, J_{\mathrm{sc}}$, curve factor (CF) and efficiency $(\eta)$ is shown in figure 9 and that on $J_{02}$ and $n_{2}$ is shown in figure 10.

Owing to the effect of GBS on the resistivity $\rho$ of polysilicon and minority carrier lifetime, as discussed earlier, the optimal $\rho$ value is relatively higher than for single crystalline cells. A comparison of the efficiencies of single and polysilicon cells as a function of $\rho$ is shown in figure 11 (Kishore 1983).

2.3b Photoconductive properties: Figure 12 depicts a light-induced change in the conductance $v$ s the position of a $\mathrm{He}-\mathrm{Ne}$ laser spot on a polysilicon wafer for three different illumination intensities of the laser (Hwang et al 1981). In this figure it is noted that the conductance at $\mathrm{GB}$ is higher than in the grain and this difference is greater for higher intensity of the laser spot. This is indeed a typical behaviour of all polysilicon wafers with electrically active GBS.

Figure 13 shows the optical picture of a polysilicon solar cell (upper part of the figure) and the response of the cell for $\lambda=9200 \AA$ scan (lower part of the figure) (Roy 1980). We see that there is a dip in the response at GB. This is a typical behaviour for all polysilicon cells and shows that the concentration of the photogenerated excess minority carriers present at $G B$ is less than in the grains. This in conjunction with the enhanced photoconductance at $G B$ as noted in figure 12 reveals that unlike the case of single crystal silicon the photoconductivity behaviour in polysilicon is not necessarily a minority carrier or more appropriately the excess carrier phenomenon.

Hwang et al (1981) and Kishore (1983) have shown that in polysilicon the low level photoconductivity is mainly due to increase in the majority carrier mobility under illumination and is therefore a majority carrier phenomenon. The increase in mobility is due to the fact that the grain boundary potential decreases under illumination. The decrease of $\phi_{B}$ with light intensity was studied for the first time theoretically by Card and Yang (1977). Experimental studies on the effect of illumination on $\phi_{B}$ have been made by Seager (1981) and Kishore (1983).

The decrease in $\phi_{B}$ under illumination leads to a large decrease in the resistivity $(\rho)$ of polysilicon. The decrease is more, if the grain size is small, the dark value of $\phi_{B}$ is high and the temperature of the polysilicon specimen is lower. Figure 14 depicts the effect of illumination on the resistivity $v$ s $1 / T$ curve for a polysilicon specimen (Kishore 1983). The decrease in $\rho$ is observed to be higher for lower values of $T$.

\subsection{Evaluation of the electrical activity of grain boundary}

The electrical activity of a GB depends on the density of interface states $\left(N_{\text {is }}\right)$ and the energy distribution of these states in the band gap. However, its effectiveness also 


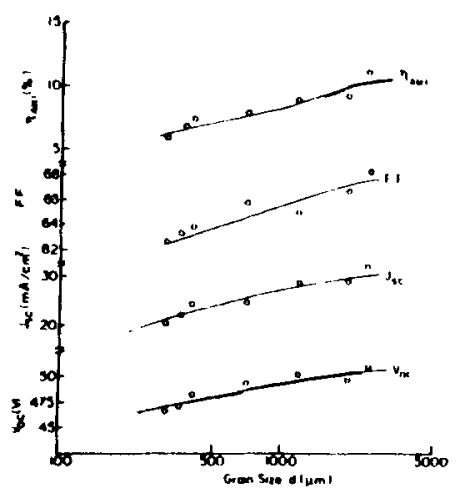

Figure 9. Plots of $V_{\text {oc }}, J_{\text {sc }}, \mathrm{CF}$ and eificiency $\eta$ as functions of grain size for solar grade polysilicon solar cells (after Kumari et al 1981).

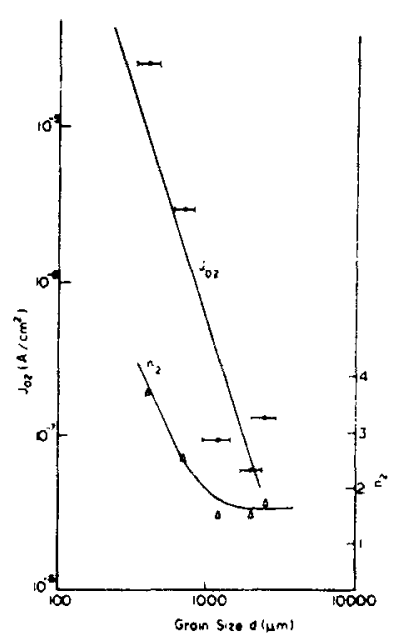

Figure 10. The effect of grain size on $J_{02}$ and $n_{2}$ for polysilicon solar cells (after Kumari et al 1981).

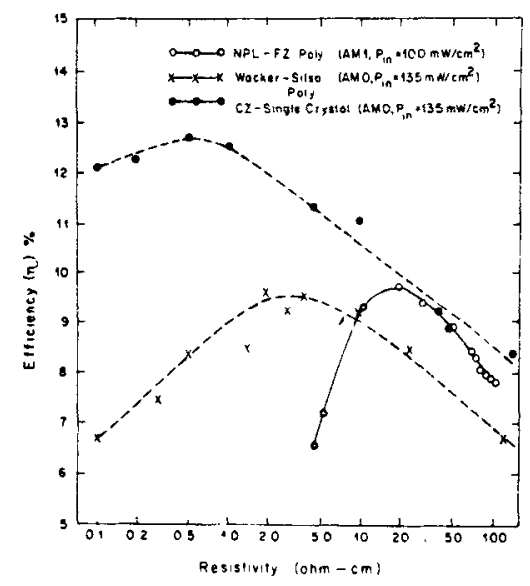

Figure 11. The conversion efficiency $\eta$ as a function of base resistivity of single and polycrystalline solar cells. (after Kishore 1983).

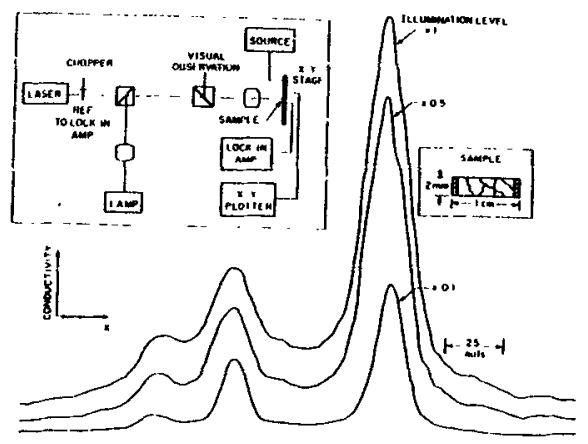

Figure 12. Light induced change in the conductance $v$ s position of a He-Ne Laser spot on a polysilicon wafer for three different intensities (after Hwang et al 1981).

depends on a number of other parameters $e g$, the doping concentration in the grain, the grain size, the Fermi level in the GB, the temperature of the specimen and also the intensity of the light in case the specimen is under illumination. The GB potential $\phi_{B}$ is directly related to the electrical activity of the GB. Therefore, the methods employed for the measurement of the electrical activity of $\mathrm{GB}$ essentially measure the quantities directly related with $\phi_{B}$. Zero bias high frequency capacitance (ZBHFC) method has been successfully used (Pike and Seager 1981) for the measurement of $\phi_{B}$ under dark and 


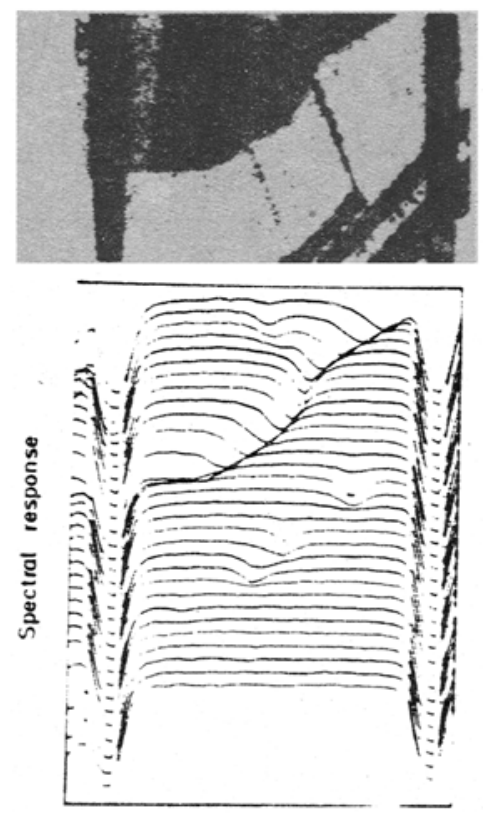

Figure 13. Spectral response of a solar cell at $920 \mathrm{~nm}$ in comparison with the optical picture of a solar cell surface. upper part: Optical picture lower part: Electrical response of the solar cell (after Roy 1980).

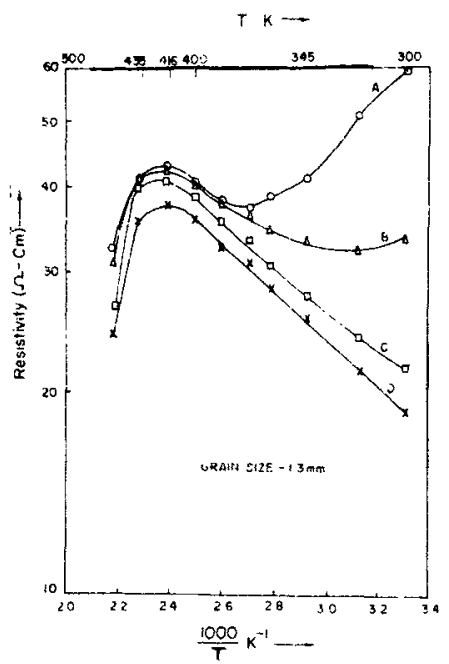

Figure 14. The effect of intensity of illumination on the temperature dependence of resistivity of a polysilicon specimen. A. for dark conditions B-D. for 30,50 and $130 \mathrm{~mW} / \mathrm{cm}^{2}$ intensity respectively. (after Kishore 1983).

illumination conditions. $\phi_{B}$ is related to the ZBHFC according to the equation

$$
\phi_{B}=q^{2}{ }_{E} N_{D} / 8 C_{H F}
$$

where $C_{H F}$ is the ZBHFC per unit area. As discussed by Lal et al (1982) this method requires the $\mathrm{GB}$ depletion layer area to be known and is thus suitable only for the specimens (eg. bicrystals) where this can bc easily determined. Pike and Seager (1981) have used this method for the measurement of $\phi_{B}$ as a function of $T$ under dark conditions and Seager (1981) has used it for determining $\phi_{B}$ as a function of the light intensity.

The $\phi_{B}$ value can also be calculated from the activation energy $\left(E_{\sigma}\right)$ of zero bias conductance (ZBC) (Pike and Seager 1981) or zero bias resistivity (ZBR) (Seager and Castner 1978) provided that $\bar{c}$ as a function of $T$ is known. The validity of these methods is limited to cases where the grain size is small so that (17) is applicable. $\phi$ having been determined $N_{\text {is }}$ can be calculated using (3) or (4), whichever may be applicable.

Recently some workers [Cheng and Shyu 1981; Shyu and Cheng 1982] have used deep level transient spectroscopy (DLTS) to identify the level location of GB states. Nonseparable nature of the GB levels and the likely presence of impurities in the GBs make the DLTS spectra complex and the identification of $\mathrm{GB}$ levels difficult.

In boron-doped bicrystals Shyu and Cheng (1982) found $G B$ level to be at $E_{v}$ $+0.38 \mathrm{eV}$ in one sample and at $E_{v}+0.48 \mathrm{eV}$ in the other. Application of DLTs may be expected to provide valuable information about the $G B$ level location in $n$ and $p$ type 
silicon and the effects of heat treatment and hydrogen passivation on the electrical activity of GBS.

\section{Polycrystalline zinc oxjde ceramic}

Polycrystalline zinc oxide, when suitably doped with various oxides like $\mathrm{Bi}_{2} \mathrm{O}_{3}, \mathrm{Sb}_{2} \mathrm{O}_{5}$, $\mathrm{CoO}, \mathrm{MnO}$, etc. exhibits voltage-dependent resistance. Since the discovery of this varistor action by Matsuka [1971], these materials have attracted the attention of various workers (Morris 1973, 1976; Levinson and Philipp 1975; Clarke 1978; Kingery et al 1979). The current $(I)$ through a varistor at a voltage $(V)$ is given by

$$
I=K V^{n}=(V / C)^{n},
$$

where $n$ is the nonlinearity exponent and $C$ is the nonlinear resistance. The nonlinearity coefficient for various types of varistors is shown in figure 15 . It can be seen that the performance of the zinc oxide varistor is comparable to that of a zenerdiode.

Zinc oxide varistors are made by sintering highly conducting grains of $\mathrm{ZnO}$ in the presence of a few mole percent of other metal oxides. The grain size depends upon the

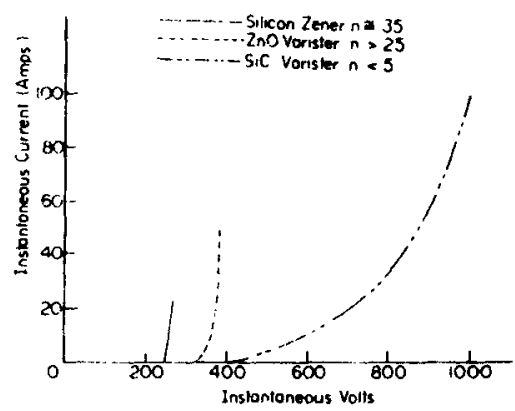

Figure 15. Instantaneous current of silicon zener, zinc oxide varistor and silicon carbide varistor vs their instantaneous voltage.

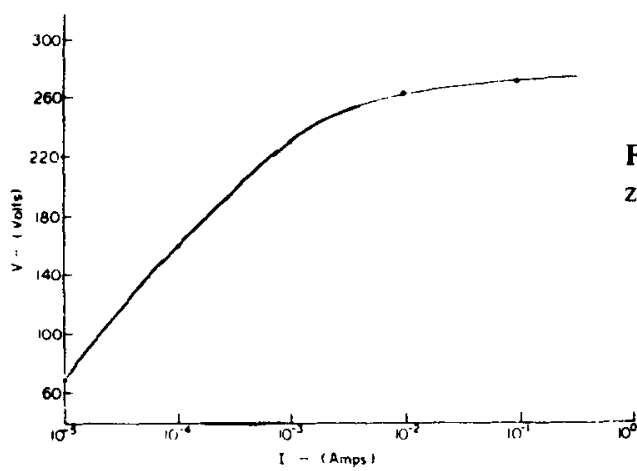

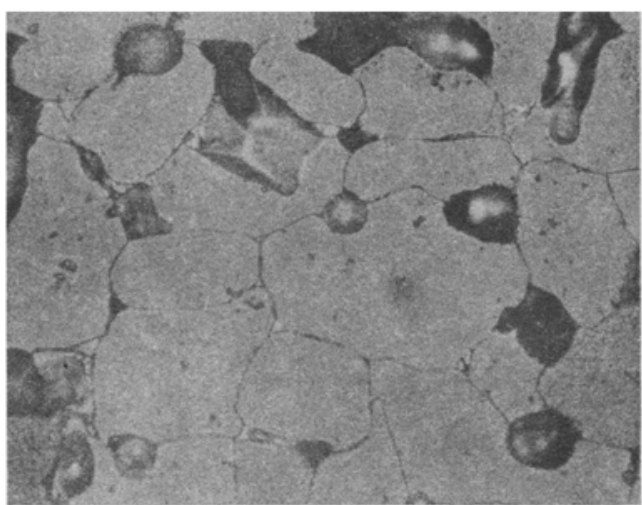

Figure 16. Typical microstructure of a zinc oxide varistor containing $6 \%$ additives.

Figure 17. Voltage is current characteristics of a zinc oxide varistor. 
initial particle size and the ceramic processing. The grains are made semiconducting by doping with a suitable metal oxide. They are $n$-type with a donor density of about $10^{17}-10^{19}$ per $\mathrm{cm}^{3}$ and show a resistivity of $1 \mathrm{ohm} \mathrm{cm}$. Figure 16 shows the microstructure of a $\mathrm{ZnO}$ varistor. Figure 17 shows an $\mathrm{I}-\mathrm{V}$ characteristic of a $\mathrm{ZnO}$ varistor prepared from $94 \mathrm{~mol} \% \mathrm{ZnO}$ and $6 \mathrm{~mol} \%$ of other additives such as $\mathrm{Bi}_{2} \mathrm{O}_{3}$, $\mathrm{CoO}, \mathrm{Sb}_{2} \mathrm{O}_{3}, \mathrm{Cr}_{2} \mathrm{O}_{3} \mathrm{MnO}$. The variation of various varistor parameters with preparation conditions is shown in figure 18.

\subsection{Grain boundaries in zinc oxide}

The unusual electrical properties of these zinc oxide varistors are due to the presence of GBS. To understand the mechanism of varistor action, the GBS of these ceramics have been intensively investigated.

Earlier theories were built on a model as given in figure 19. It conceived the material as consisting of two phases-one consisting of an insulating GB phase (probably a glassy phase) coating the GB. This was based on optical and SEM observations. The intergranular layer was believed to be several hundred Angstroms thick.

However detailed examination of the GB region by STEM showed that there is no discrete intergranular phase at the boundary between two grains although an interconnected phase exists at three and four grain corners (Santhanam et al 1979). Using high resolution $\mathrm{x}$-ray micro analysis it was shown that the GBs are enriched with bismuth [Clarke 1978; Kingery et al 1979]. A model to explain the varistor action should be based on the concentration profile of segregating atoms at the GBS.

\subsection{Origin of varistor action}

Earlier, it was thought that the nonlinear volt-ampere characteristic of the $\mathrm{ZnO}$ ceramic was intimately connected with the conduction mechanism in the intergranular

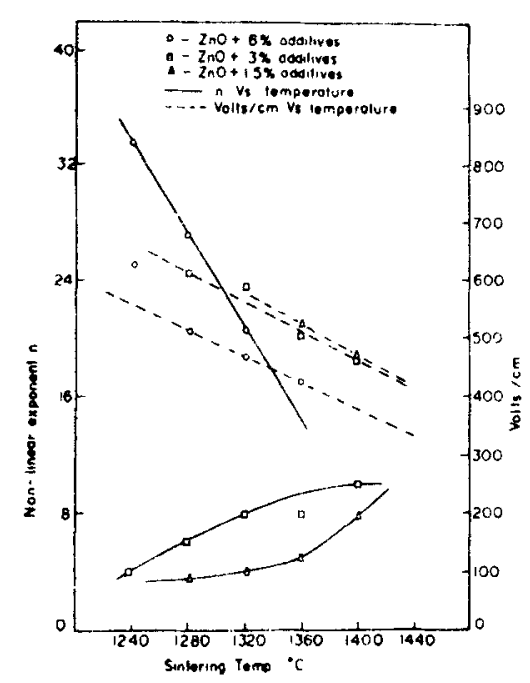

Figure 18. Variation of varistor parameters with sintering temperature (after Kalsi and Das 1983).

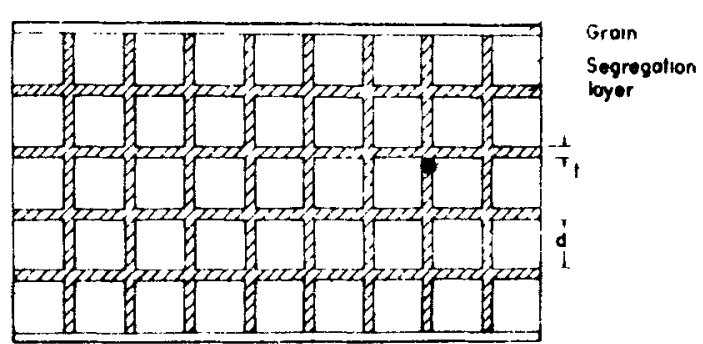

Figure 19. Schematic diagram of microstructure of zinc oxide ceramics. 
phase. On the basis of electrical properties measured on $\mathrm{ZnO}-0.5 \mathrm{~mol} \% \mathrm{Bi}_{2} \mathrm{O}_{3}$ by Morris (1973), the author proposed a combination of electron hopping and tunneling in the thin layers of amorphous $\mathrm{Bi}_{2} \mathrm{O}_{3}-\mathrm{ZnO}$, as the model to explain varistor action in this material. The author observed the segregation of $\mathrm{Bi}_{2} \mathrm{O}_{3}$ amorphous phase at the GBS. He estimated a thickness of $\simeq 600 \AA$ for the intergranular phase which is large for tunneling through it to occur. He then assumed a process consisting of a series of tunneling and hopping steps (1982).

Later by direct measurements Einzinger emphasized that the presence of heterogeneous segregation layers between $\mathrm{ZnO}$ grains was no basic requirement for the varistor effect. According to the author the effect was located in the vicinity of the directly touching $\mathrm{ZnO}$ grains and was therefore assumed to be a property of doped $\mathrm{ZnO}$.

Therefore the theories, which depended upon the existence of thick intergranular region are now obsolete. According to the recent theories (Mahan 1982), the important intergranular regions, for the nonlinear current properties, are composed of one or two atomic layers. Due to the trapping of excess electrons at the intergranular region, a Schottky barrier is formed, adjacent to the interface, in each $\mathrm{ZnO}$ grain. The situation becomes similar to the one described for polysilicon as discussed under $\S 2$.1c. There is much experimental evidence for the existence of this double Schottky barrier model (figure 20). The nonlinear electrical behaviour is caused by electrons trying to get past this barrier. Electron transport through the interface is believed to be a two-step process, whereby first the electron goes from $\mathrm{ZnO}$ grain to the interface and secondly on to the next $\mathrm{ZnO}$ grain.

This simple model is successful in explaining the current to be thermally activated with an activation energy equal to the barrier height $\phi_{B}$ (Mahan 1982). The double Schottky barrier in fact behaves as a single barrier at reverse bias as is obvious from the capacitance measurements.

$$
C^{-2} \propto\left(\phi_{B}+V\right) \text {. }
$$

An improvement in this model was proposed to explain the pre-breakdown current (Mahan et al 1978, 1979). According to them, at higher electric fields, the reverse biased depletion region becomes very thin and electron tunnelling should be taken into account. The current is then dominated by tunnelling through the depletion region,

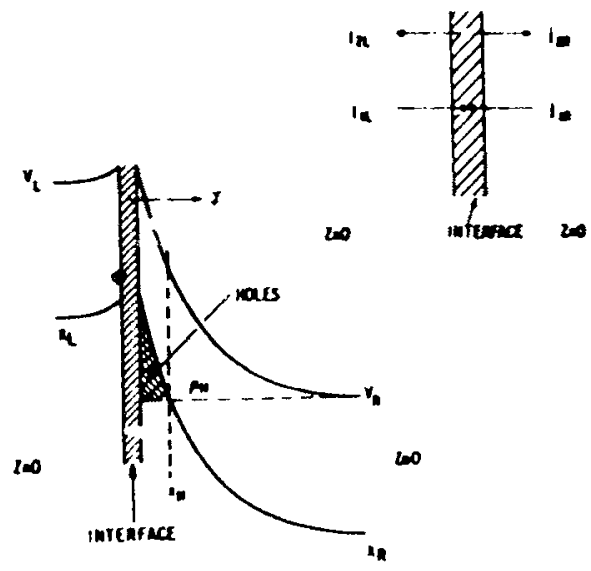

Figure 20. The double Schottky barrier model, showing depletion regions on two sides. The holes only exist after breakdown. The inset shows the four tunneling components in two step transport (after Mahan et al 1979). 
rather than thermal excitation over it. The inclusion of this mechanism provides excellent agreement between theory and experiment for the pre-breakdown current.

Various models have been proposed to explain the breakdown in $\mathrm{ZnO}$ varistors. Zener tunnelling was suggested as the mechanism (Bernasconi et al 1976). Another theory assumes that the breakdown occurs because the barrier heights vanish (Levine 1975; Bernasconi et al 1976). Without barriers, the GB no longer offers any resistance. But this theory has not been successful in explaining the sharp rise in capacitance at breakdown.

Mahan et al (1978) proposed that breakdown occurred because holes are created in the $\mathrm{ZnO}$ valence band, by the excitation of electron hole pairs. These electron hole pairs are excited by the electrons entering the $\mathrm{ZnO}$ conduction band. The holes get trapped in the depletion region, providing additional positive charge which makes the depletion region much thinner. The electrons can then easily tunnel through the depletion region. This theory explains the sharp rise in capacitance at the breakdown voltage.

All these theories have used a single GB to describe the nonlinear electrical behaviour. Actual macroscopic devices present the additional problem of a random network of GBS, each with nonlinear behaviour. Little or no progress has yet been made on the macroscopic system of random networks. However, the experiments show that a single GB has the nonlinear electrical properties, and the network is thought to be multistages of the same nonlinear element.

\section{References}

Baccarani G, Ricco B and Spadini G 1978 J Appl. Phys. 495565

Bernasconi J, Klein H P, Knecht B and Strassler S J 1976 Electr. Mater. 5473

Card H C and Yang E S 1977 IEEE Trans. Electron Devices ED-24 397

Cheng L J and Shyu C M 1981 Semiconductor silicon (ed.) H R Huff R J Kriegler and Y Takeishi (Pennigton New Jersey: The Electrochemical Society) 390

Clarke D R 1978 J. Appl. Phys. 492407

Colinge J P, Demoulin E, Delannay F, Lobet M and Temerson J M 1981 J. Electrochem. Soc. 1282009

Crowell C R and Sze S M 1966 Solid State Electron. 91035

Einzinger R 1982 Grain boundaries in semiconductors (eds) H J Leamy G E Pike and C H Seager (New York: North Holland Publn Co.) 343

Fischer H and Pschunder P 1977 IEEE Trans. Electron Devices ED-24 438

Fossum J G and Lindholm F A 1980 IEEE Trans. Electron Devices ED-27 692

Gaef M W M, Bloem J, Giling L J, Monkowski J R and Maes J W C 1979 2nd E C photovoltaic solar energy conf. Berlin p. 65

Hwang W, Panayotatos P, Poon E and Card H C 1981 Proc. 15th IEEE Photovoltaic specialists' Conf. 871 Jain G C, Chakravarty B C and Singh S N 1981 Appl. Phys. Lett. 38815

Jain G C, Singh S N and Kumari S 1980a Proc. 3rd E C Photovoltaic solar energy conf. Cannes, France Jain G C, Singh S N, Arora N K and Das B K 1980 b Proc. national solar energy convention Annamalai Nagar, India 317

Kalsi H S and Das B K 1983 Bull. Mater. Sci. 533

Kazmerski L L, Ireland P J and Ciszek T F 1980 Appl. Phys. Lett. 36323

Kim D M, Khondker A N, Shah R R and Crosthwait D L 1981 IEEE Electron Device Lett. Vol EDL-3141

Kingery W D, Vander Sande J B and Mitamura T 1979 J Am. Ceram. Soc. 62221

Kishore R 1983 Effect of various material parameters on the performance of silicon solar cells $\mathrm{Ph}$. D Thesis, Agra University, Agra

Kishore R, Singh S N and Singh P K 1983 Proc national solar energy convention, Vadodara, India (to be published)

Kumar K R and Satyam M 1981 Appl. Phys. Lett. 39898

Kumari S, Arora N K and Jain G C 1981 Sol. Energy Mater. 5383 
Kumari S, Jain S K, Das B K and Jain G C 1983 Sol. Cells 9209

Lal Mohan, Kishore R, Singh S N and Das B K 1982 Proc. National Solar Energy Convention, Delhi, India 9.040

Levine J D 1975 CRC Crit. Rev. Solid State Sci. 5597

Levinson L M and Philipp H R 1975 J. Appl. Phys. 461332

Lindmayer J 1978 Proc. 13th IEEE Photovoltaic specialists, conf. p. 1096

Mahan G D, Levinson L M and Philipp H R 1978 Appl. Phys. Lett. 33830

Mahan G D, Levinson L M and Philipp H R 1979 J. Appl. Phys. 502799

Mahan G D 1982 Grain boundaries in semiconductors (ed) H J Leamy, G E Pike and C H Seager (New York: North Holland Pubin Co.) 333

Martinez J and Piqueras J 1980 Solid State Electron. 23297

Matre H F 1971 Defect electronics in semiconductors (New York: Wiley Interscience)

Matsuka M 1971 Jpn. J. Appl. Phys. 10736

Morris W G 1973 J. Am. Ceram. Soc. 56360

Morris W G 1976 J. Vac. Sci. Tech. 13926

Pike G E and Seager C H 1981 Adv. Ceram. 183

Pollock G A, Deline V R and Furman B K 1982 Grain boundaries in semiconductors (ed) H J Leamy, G E Pike and C H Seager (New York: North Holland Publn Co.) 71

Poon E and Hwang W 1982 Solid State Electron. 25699

Roy K 1980 Proc. symp. on electrical and optical properties polycrystalline or impure semiconductors and novel silicon growth methods (eds) K V Ravi and B O'Mara (Pennigton New Jersey: The Electrochemical Society) (eds) 1

Santhanam A T, Gupta T K and Carlson W G 1979 J. Appl. Phys. 50852

Seager C H 1981 J. Appl. Phys. 523960

Seager C H and Castner T G 1978 J. Appl. Phys. 493879

Seto J Y W 1975 J. Appl. Phys. 465247

Shyu C M and Cheng C J 1982 Grain boundaries in semiconductors (ed) H J Leamy, G E Pike and C H Seager (New York: North Holland Publn Co.) 131 